\title{
Brief paper \\ Active surge control of compression system using drive torque
}

\author{
Bjørnar Bøhagen ${ }^{\mathrm{a}, *}$, Jan Tommy Gravdahl ${ }^{\mathrm{b}}$ \\ ${ }^{\mathrm{a}} \mathrm{EOP}, \mathrm{ABB}$, Oslo, Norway \\ ${ }^{\mathrm{b}}$ Department of Engineering Cybernetics, Norwegian University of Science and Technology, Trondheim, Norway
}

Available online 21 February 2008

\begin{abstract}
Novel control laws for a compression system using only the drive torque as control input are presented. Stabilization of any desired operating point is achieved with static or dynamic feedback. The stability results are derived using backstepping and passivity techniques.
\end{abstract}

(C) 2007 Published by Elsevier Ltd.

Keywords: Compressor; Active surge control; Nonlinear

\section{Introduction}

Towards low mass flows, the stable operating region of centrifugal compressors is bounded due to the occurrence of surge. This phenomenon is characterized by oscillations in system states such as pressure and mass flow. This is undesirable since it introduces the possibility of severe damage to the machine due to vibrations and high thermal loading resulting from lowered efficiency.

Compressor performance is usual described using a compressor map as in Fig. 1. This map describes the relation between compressor pressure ratio, mass flow and speed, using constant speed lines in a flow-pressure coordinate system. Surge is an unstable operational mode of the compressor and the stability boundary in the compressor map is called the surge line. This line divides the compressor map in two regions, where the region to the left and right of the surge line corresponds to open loop stable and unstable operation, respectively.

Traditionally, compressors have been controlled by using surge avoidance schemes. Such schemes use various means in order to keep the operating point of the machine away from the region where surge occurs. Typically, a surge control line is drawn at a distance from the surge line, leaving a surge margin

\footnotetext{
This paper was not presented at any IFAC meeting. This paper was recommended for publication in revised form by Associate Editor Reza Katebi under the direction of Editor Mituhiko Araki.

* Corresponding author.

E-mail addresses: Bjornar.Bohagen@no.abb.com (B. Bøhagen), Tommy.Gravdahl@itk.ntnu.no (J.T. Gravdahl).
}

in the compressor map. The surge avoidance scheme then ensures operation to the right or at the surge control line, see Fig. 1. This method restricts the operating range of the machine to the region in which the system is open loop stable, resulting in limited efficiency and operational range.

Active surge control is fundamentally different from surge avoidance. In an active surge control scheme the open loop unstable region of the compressor map is sought stabilized with feedback rather than avoided. Thus, the possible operating regime of the machine is enlarged. This approach was first introduced by Epstein, Ffowcs Williams, and Greitzer (1989), and since then a number of results have been published. Different actuators have been used and examples include recycle, bleed and throttle valves, gas injection, variable guide vanes and drive torque. For an overview consult Gravdahl and Egeland (1999), van de Wal (1998), and Willems and de Jager (1999).

In this paper we propose control schemes using only the drive torque to actively stabilize a compression system. The idea was initially introduced in Gravdahl, Egeland, and Vatland (2002), and further pursued in Bøhagen and Gravdahl $(2005,2006)$.

\section{Model}

A classical result in the field of compressor surge modeling is the model of Greitzer (1976), which covers a basic compression system consisting of a compressor, a plenum volume, a throttle and in-between ducting. In Fink, Cumpsty, and Greitzer (1992) the authors extended the Greitzer model to also 


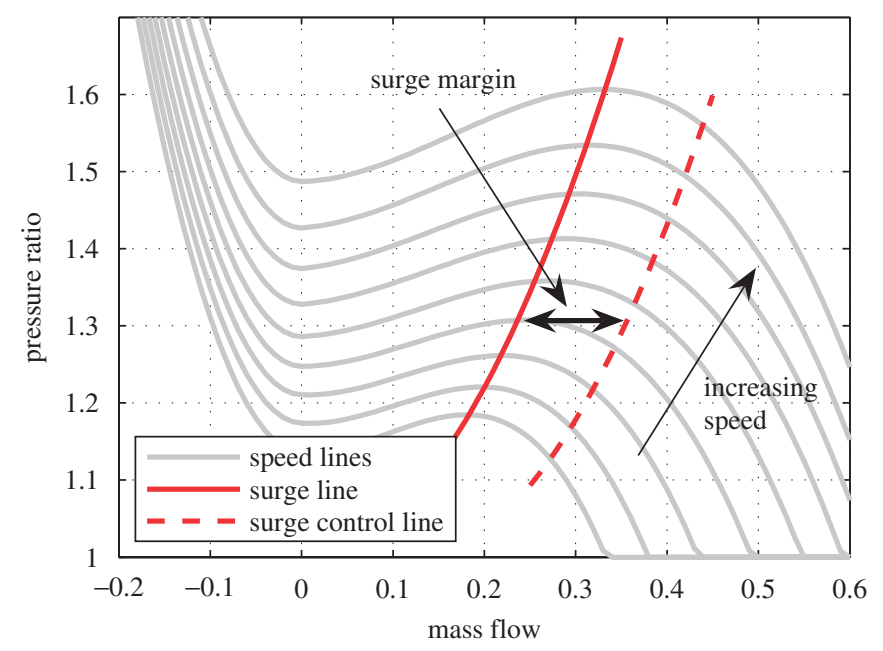

Fig. 1. Compressor map and related definitions.

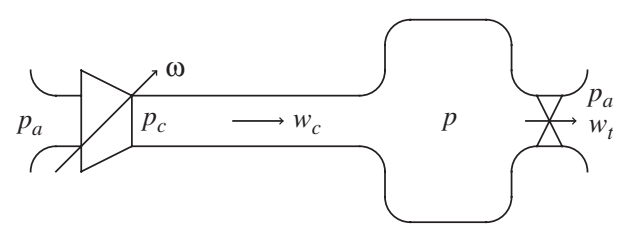

Fig. 2. Compression system.

incorporate variable impeller speed, in addition to duct mass flows and plenum pressure. A similar model was derived in Gravdahl and Egeland (1999), which is the model to be used here.

Consider a compression system working between two pressure reservoirs, as illustrated in Fig. 2. A centrifugal compressor supplies compressed gas to a duct which discharges into a plenum volume, from which the compressed gas discharges over a throttle. Plenum pressure dynamics is derived by evaluating the mass balance of the plenum volume, assuming isentropic conditions, ideal gas and uniform pressure. Duct mass flow dynamics is derived by evaluating the momentum balance of the duct connecting compressor and plenum assuming incompressible one-dimensional flow in the duct. Furthermore, dynamic effects related to the compressor stage are assumed small, leaving pressure downstream the compressor as a pure mapping of mass flow and impeller speed. Impeller speed dynamics is derived by evaluating the angular momentum balance of the rotating parts. This is where the drive torque appears, assumed to be at our disposal as system input. Following Gravdahl and Egeland (1999), the resulting model, which is experimentally validated in Gravdahl, Willems, de Jager, and Egeland (2004), for this system is given by

$\dot{p}=\frac{c^{2}}{V}\left(w-w_{\mathrm{t}}(p)\right)$,

$\dot{w}=\frac{A}{L}\left(p_{\mathrm{c}}(w, \omega)-p\right)$,

$\dot{\omega}=\frac{1}{J}\left(\tau_{d}-\tau_{c}(w, \omega)\right)$, where $p$ is plenum pressure, $w$ is duct mass flow and $\omega$ is impeller speed. Mass flow through the throttle is given by $w_{\mathrm{t}}(p)$, pressure downstream compressor is denoted $p_{\mathrm{c}}(w, \omega)$ and load torque on the impeller due to compressor fluid flow is given by $\tau_{c}(w, \omega)$. Furthermore, $c$ is the speed of sound at ambient conditions, $V$ is the volume of plenum, $A$ is the duct cross section of duct, $L$ is the length of duct and $J$ is inertia of rotating parts. Models for throttle mass flow and compressor torque are given by

$w_{\mathrm{t}}(p)=k_{\mathrm{t}} \operatorname{sign}\left(p-p_{\mathrm{a}}\right) \sqrt{\left|p-p_{\mathrm{a}}\right|}$,

$\tau_{c}(w, \omega)=\sigma r^{2}|w| \omega$

where $k_{\mathrm{t}}$ reflects the throttle opening, $p_{\mathrm{a}}$ is the ambient pressure, $\sigma$ is a slip factor and $r$ is impeller radius. The model for throttle mass flow is slightly different from Gravdahl and Egeland (1999), allowing reverse flow also for this component. To this extent the throttle flow is assumed to be symmetric with respect to pressure difference. Pressure downstream the compressor has the same qualitative form as the map shown in Fig. 1, but an explicit expression will not be given here. A model for this pressure can be derived from fit of experimental data, e.g. polynomial Willems (2000), or be based on theoretical models Gravdahl et al. (2004). Furthermore, it is known that pressure downstream the compressor increases with increasing impeller speed.

Fact 1. Pressure downstream the compressor, $p_{\mathrm{c}}(w, \omega)$, is strictly increasing with increasing impeller speed, $\omega$. Furthermore, it is assumed that $\partial p_{\mathrm{c}}(w, \omega) / \partial w$ is upper bounded.

The control laws to be derived do not depend on a specific structure for the mapping $p_{\mathrm{c}}(w, \omega)$, but rather on an overall property related to its gradients. More specifically, we will require for one of the control gains, say $c$, that

$c \geqslant \sup \left\{\frac{\frac{\partial p_{\mathrm{c}}(w, \omega)}{\partial w}+\delta}{\frac{\partial p_{\mathrm{c}}(w, \omega)}{\partial \omega}}\right\}$,

where $\delta$ is some arbitrary small positive constant. From Fact 1 it is known that the impeller speed gradient is strictly positive and the compressor map shows that the mass flow gradient is positive only between zero mass flow and surge line. This implies that $c$ must be positive. Furthermore, these observations suggest that $c$ is bounded.

From (1)-(5) it can be seen that a equilibrium, $(\cdot)^{\mathrm{e}}$, satisfy $w^{\mathrm{e}}=k_{\mathrm{t}} \sqrt{p^{\mathrm{e}}-p_{\mathrm{a}}}, p_{\mathrm{c}}\left(w^{\mathrm{e}}, \omega^{\mathrm{e}}\right)=p^{\mathrm{e}}$ and $\tau_{d}^{\mathrm{e}}=\sigma r^{2} w^{\mathrm{e}} \omega^{\mathrm{e}}$ when for all practical cases the desired equilibrium involves positive mass flow. For notational convenience of subsequent sections we let $(\bar{\cdot})=(\cdot)-(\cdot)^{\mathrm{e}}$ denote deviation from a desired equilibrium.

\section{Control laws}

In this section control laws to asymptotically stabilize a desired equilibrium of (1)-(3) are presented, when considering 
$\tau_{d}$ as input. The control inputs are based on a backstepping approach presented in Section 4.

Proposition 2. Either of the control inputs $\tau_{d}=\tau_{a}$ or $\tau_{d}=\tau_{b}$, where

$$
\begin{aligned}
\tau_{a}= & -c_{4}\left(\bar{\omega}+c_{3} \bar{w}\right)+c_{3} \frac{A J}{L}\left(p_{\mathrm{c}}(w, \omega)-p\right)+\sigma r^{2}|w| \omega \\
\tau_{b}= & -c_{4}\left(\bar{\omega}+c_{3} \bar{w}\right)+c_{3} \frac{A J}{L}\left(p_{\mathrm{c}}(w, \omega)-p\right) \\
& +\sigma r^{2}|w|\left(\omega^{\mathrm{e}}-c_{3} \bar{w}\right),
\end{aligned}
$$

will make the desired equilibrium globally asymptotically stable when $c_{3}$ is chosen according to (6) and $c_{4}>0$.

\section{Proof. See Section 4}

The next proposition extends the control laws by adding a passive control part $\tau_{\text {passive }}$, which can be either static or dynamic. For the static part we consider $\tau_{\text {passive }}=\psi\left(t, u_{\tau}\right)$ where $u_{\tau}=\bar{\omega}+c_{3} \bar{w}$ and for the dynamic part we consider a system $\Sigma_{\text {passive }}$ defined by $\dot{\tau}=f\left(\tau, u_{\tau}\right)$ and $\tau_{\text {passive }}=h\left(\tau, u_{\tau}\right)$, where $u_{\tau}$ and $\tau_{\text {passive }}$ are system input and output, respectively.

Proposition 3. Either of the control inputs $\tau_{d}=\tau_{a}-\tau_{\text {passive }}$ or $\tau_{d}=\tau_{b}-\tau_{\text {passive }}$ where $\tau_{a}$ and $\tau_{b}$ as in Proposition 2, and

- $\tau_{\text {passive }}$ is given by the time-varying memoryless passive function $\psi$, will make the desired equilibrium globally asymptotically stable.

- $\tau_{\text {passive }}$ is given by the strictly passive or output strictly passive and zero state observable system $\Sigma_{\text {passive for which a }}$ positive definite and radially unbounded storage function exists, will make the desired equilibrium globally asymptotically stable.

- $\tau_{\text {passive }}$ is given by the passive system $\Sigma_{\text {passive }}$ for which a positive definite and radially unbounded storage function exists, will make system states converge the desired equilibrium.

Proof. See Section 4.

From Proposition 2 we recognize the term $-c_{4} u_{\tau}$ for both implementations. This term can be "replaced" by a saturated alternative by choosing $c_{4}$ vanishingly small and implementing the passive part as $\tau_{\text {passive }}=\operatorname{sat}\left(c_{4}\right.$,passive $\left.u_{\tau}\right)$.

\section{Proofs}

By a closer inspection of the model (1)-(3), it is clear that it is in a pure-feedback form, Krstić, Kanellakopoulos, and Kokotović (1995). It is prevented from being in strict-feedback form since $\omega$ do not appear affine in (2), but through the mapping $p_{\mathrm{c}}(w, \omega)$. The practical implication of this is that stepping back on $\omega$ becomes somewhat more complicated. Using backstepping to stabilize the compressions system was also done in Krstić, Fontaine, Kokotović, and Paduano (1998), where flow through the throttle was used as control input.
Using (1)-(3) the system can be expressed in error variables

$\dot{\bar{p}}=k_{1}\left(\bar{w}+f_{1}(\bar{p})\right)$

$\dot{\bar{w}}=k_{2}\left(f_{2}(\bar{w}, \bar{\omega})-\bar{p}\right)$,

$\dot{\bar{\omega}}=k_{3}\left(u+f_{3}(\bar{w}, \bar{\omega})\right)$,

where

$f_{1}(\bar{p})=w^{\mathrm{e}}-w_{\mathrm{t}}\left(\bar{p}+p^{\mathrm{e}}\right)$,

$f_{2}(\bar{w}, \bar{\omega})=p_{\mathrm{c}}\left(\bar{w}+w^{\mathrm{e}}, \bar{\omega}+\omega^{\mathrm{e}}\right)-p^{\mathrm{e}}$,

$f_{3}(\bar{w}, \bar{\omega})=\tau_{d}^{\mathrm{e}}-\tau_{c}\left(\bar{w}+w^{\mathrm{e}}, \bar{\omega}+\omega^{\mathrm{e}}\right)$,

$u=\tau_{d}-\tau_{d}^{\mathrm{e}}$

and constants are given by $k_{1}=c^{2} / V, k_{2}=A / L$ and $k_{3}=1 / J$.

Remark 4. From (10)-(12), (4)-(5) and Fact 1 it can be recognized that

$(a-b)\left(f_{1}(a)-f_{1}(b)\right)<0$,

$(a-b)\left(f_{2}(\bar{w}, a)-f_{2}(\bar{w}, b)\right)>0$,

$(a-b)\left(f_{3}(\bar{w}, a)-f_{3}(\bar{w}, b)\right) \leqslant 0$,

and $f_{1}(0)=f_{2}(0,0)=f_{3}(0,0)=0$.

The design starts by defining $z_{1}=\bar{p}$ as the first backstepping variable and considering $\bar{w}$ of (7) as virtual control. This introduces the second variable $z_{2}=\bar{w}-\alpha_{2}\left(z_{1}\right)$ where $\alpha_{2}$ is the related stabilizing function. The time derivative of $V_{1}\left(z_{1}\right)=\frac{1}{2} d_{1} z_{1}^{2}$ is then expressed in terms of $z_{1}$ and $z_{2}, \dot{V}_{1}\left(z_{1}\right)=d_{1} k_{1} z_{1} f_{1}\left(z_{1}\right)+$ $d_{1} k_{1} z_{1} z_{2}+d_{1} k_{1} z_{1} \alpha_{2}\left(z_{1}\right)$, where it is known from Remark 4 that $z_{1} f_{1}\left(z_{1}\right)$ is negative definite in $z_{1}$. This implies that $\alpha_{2}$ is not absolutely needed to introduce a term to make $\dot{V}_{1}$ negative definite in $z_{1}$. Even if a choice different from $\alpha_{2}\left(z_{1}\right)=0$ might be beneficial later in the design, it is preferred to keep the structural complexity of the controller low.

The second backstepping variable, $z_{2}=\bar{w}$, then considers $\bar{\omega}$ of (8) as virtual control. This introduces the third error variable $z_{3}=\bar{\omega}-\alpha_{3}\left(z_{1}, z_{2}\right)$, where $\alpha_{3}$ is the related stabilizing function. The time derivative of $V_{2}\left(z_{1}, z_{2}\right)=$ $V_{1}\left(z_{1}\right)+\frac{1}{2} d_{2} z_{2}^{2}$ is then expressed in terms of $z_{1}, z_{2}$ and $z_{3}, \quad \dot{V}_{2}\left(z_{1}, z_{2}\right)=d_{1} k_{1} z_{1} f_{1}\left(z_{1}\right)+d_{2} k_{2} z_{2} f_{2}\left(z_{2}, \alpha_{3}\left(z_{1}, z_{2}\right)\right)+$ $d_{2} k_{2} z_{2} \bar{f}_{2}\left(z_{1}, z_{2}, z_{3}\right)$ where $\bar{f}_{2}\left(z_{1}, z_{2}, z_{3}\right)=f_{2}\left(z_{2}, z_{3}+\right.$ $\left.\alpha_{3}\left(z_{1}, z_{2}\right)\right)-f_{2}\left(z_{2}, \alpha_{3}\left(z_{1}, z_{2}\right)\right)$ and the choice $d_{1}=d_{2} k_{2} / k_{1}$ has been made in order to cancel out cross terms of $z_{1} z_{2}$. The term $z_{2} f_{2}\left(z_{2}, \alpha_{3}\left(z_{1}, z_{2}\right)\right)$ is used in the same manner as Gravdahl et al. (2002), by choosing $\alpha_{3}\left(z_{1}, z_{2}\right)=-c_{3} z_{2}$ and require $c_{3}$ to satisfy (6). This gives the upper bound $z_{2} f_{2}\left(z_{2}, \alpha_{3}\left(z_{2}\right)\right) \leqslant-k_{c 3} z_{2}^{2}$, where $k_{c 3}$ is identical to $\delta$ of (6).

For the third and final backstepping variable, $z_{3}=$ $\bar{\omega}+c_{3} z_{2}$, our control input $u$ now appears. Using the upper bound on $\dot{V}_{2}$, the time derivative of $V_{3}(z)=V_{2}\left(z_{1}, z_{2}\right)+$ $\frac{1}{2} d_{3} z_{3}^{2}$ is upper bounded by $\dot{V}_{3}\left(z_{1}, z_{2}, z_{3}\right) \leqslant d_{1} k_{1} z_{1}$ $f_{1}\left(z_{1}\right)-d_{2} k_{2} k_{c 3} z_{2}^{2}+d_{2} k_{2} z_{2} \bar{f}_{2}\left(z_{2}, z_{3}\right)+d_{3} k_{3} z_{3}\left(u+f_{3}\left(z_{2}\right.\right.$, $\left.\left.z_{3}-c_{3} z_{2}\right)+c_{3}\left(k_{2} / k_{3}\right)\left(f_{2}\left(z_{2}, z_{3}-c_{3} z_{2}\right)-z_{1}\right)\right)$, where 
$\bar{f}_{2}\left(z_{1}, z_{2}, z_{3}\right)=\bar{f}_{2}\left(z_{2}, z_{3}\right)$ due to the choice of $\alpha_{3}$. Applying the mean value theorem, it can be shown that $z_{2} \bar{f}_{2}\left(z_{2}, z_{3}\right)=$ $\left(\partial f_{2}\left(z_{2}, l\right) / \partial l\right) z_{2} z_{3}$ where $l \in L\left(\alpha_{3}, z_{3}-c_{3} z_{2}\right) .{ }^{1}$ The resulting cross term $z_{2} z_{3}$ is now dominated by using the already present quadratic term in $z_{2}$ and a quadratic term in $z_{3}$ introduced by the control law

$u=u_{1}+u_{2}+u_{3} \quad$ and $\quad u_{1}=-c_{4} z_{3}$

where $c_{4}$ is a positive constant and inputs other than $u_{1}$ will be clarified in subsequent discussion. For notational convenience let $k_{c 3}=k_{c 3}^{\prime}+\delta_{2}$ and $c_{4}=c_{4}^{\prime}+\delta_{3}$, where the $\delta_{2}$ and $\delta_{3}$ are arbitrarily small positive constants. The upper bound on $\dot{V}_{3}$ is now rewritten

$$
\begin{aligned}
\dot{V}_{3}(z) \leqslant & -d_{3} k_{3} W_{3}(z)-d_{3} k_{3} z_{2,3}^{\mathrm{T}} Q(t, z) z_{2,3} \\
+ & d_{3} k_{3} z_{3} g\left(u_{2}, z\right)+d_{3} k_{3} z_{3} u_{3}, \\
g\left(u_{2}, z\right)= & u_{2}+f_{3}\left(z_{2}, z_{3}-c_{3} z_{2}\right) \\
& +c_{3} \frac{k_{2}}{k_{3}}\left(f_{2}\left(z_{2}, z_{3}-c_{3} z_{2}\right)-z_{1}\right),
\end{aligned}
$$

$W_{3}(z)=-\frac{d_{1} k_{1}}{d_{3} k_{3}} z_{1} f_{1}\left(z_{1}\right)+d \delta_{2} z_{2}^{2}+\delta_{3} z_{3}^{2}$,

$Q(t, z)=\left[\begin{array}{cc}d k_{c 3}^{\prime} & -\frac{1}{2} d \frac{\partial f_{2}\left(z_{2}, l\right)}{\partial l} \\ -\frac{1}{2} d \frac{\partial f_{2}\left(z_{2}, l\right)}{\partial l} & c_{4}^{\prime}\end{array}\right]$,

where $z=\left(z_{1}, z_{2}, z_{3}\right), z_{2,3}=\left(z_{2}, z_{3}\right)$ and $d=d_{2} k_{2} / d_{3} k_{3}$. From (17) and Remark 4 it can be recognized that $W_{3}(z)$ is positive definite in $z$. The matrix (18) is considered as a function of $t$ and $z$ since the exact point at which $\partial f_{2}\left(z_{2}, l\right) / \partial l$ is evaluated is not known, when $l$ is some point on a line segment defined by $z_{2}$ and $z_{3}$. By evaluating the upper left determinants it can be seen that $Q(t, z)>0$ when $c_{4}^{\prime} k_{c 3}^{\prime}>(d / 4)\left(\partial f_{2}\left(z_{2}, l\right) / \partial l\right)^{2}$. Choosing $d$ sufficiently small, the region of $z$ for which $Q(t, z)>0$ can be made arbitrarily large, assuming that $\partial f_{2}\left(z_{2}, l\right) / \partial l$ is bounded for bounded $z$. It follows that $Q(t, z)$ is positive definite if it can be assumed that this gradient is globally bounded. Hence, in view of the assumption in Fact 1, an upper bound on (15) is given by

$\dot{V}_{3}(z) \leqslant-d_{3} k_{3} W_{3}(z)+d_{3} k_{3} z_{3} g\left(u_{2}, z\right)+d_{3} k_{3} z_{3} u_{3}$.

Let now the control input $u_{2}$ be used to cancel all terms of $g$ and choose $u_{3}=0$, as is the case for the first equation of Proposition $2\left(\tau_{d}=\tau_{d}^{\mathrm{e}}+u_{1}+u_{2}\right)$. Then we can conclude that $z=0$ is globally asymptotically stable since $V_{3}$ is positive definite and radially unbounded in $z$ and $\dot{V}_{3}$ is negative definite in $z$. Moreover, it can be recognized that $z_{1} f_{1}\left(z_{1}\right) \leqslant-\delta_{1} z_{1}^{2}$ for some positive $\delta_{1}$ semi globally, by which it is concluded that $z=0$ is semi globally exponentially stable.

An alternative formulation for compressor torque in (16) is $f_{3}\left(z_{2}, z_{3}+\alpha_{3}\right)=f_{3}\left(z_{2}, \bar{\omega}\right)-f_{3}\left(z_{2}, \alpha_{3}\right)+f_{3}\left(z_{2}, \alpha_{3}\right)$, where it has been used that $\bar{\omega}=z_{3}+\alpha_{3}$. From Remark 4 it can be seen that $d_{3} k_{3} z_{3} g\left(u_{2}, z\right) \leqslant d_{3} k_{3} z_{3} g^{\prime}\left(u_{2}, z\right)$ where

\footnotetext{
${ }^{1} L(a, b)$ is the line segment joining the points $a$ and $b$.
}

$g^{\prime}\left(u_{2}, z\right)=u_{2}+f_{3}\left(z_{2}, \alpha_{3}\right)+c_{3}\left(k_{2} / k_{3}\right)\left(f_{2}\left(z_{2}, z_{3}+\alpha_{3}\right)-z_{1}\right)$, since $z_{3}\left(f_{3}\left(z_{2}, \bar{\omega}\right)-f_{3}\left(z_{2}, \alpha_{3}\right)\right) \leqslant 0$ and $d_{3} k_{3}>0$. Hence, an alternative upper bound for (19) is $\dot{V}_{3}(z) \leqslant-d_{3} k_{3} W_{3}(z)+$ $d_{3} k_{3} z_{3} g^{\prime}\left(\tau_{d}, z\right)+d_{3} k_{3} z_{3} u_{3}$. The second equation of Proposition 2 then results from choosing $u_{2}$ to cancel all terms of $g^{\prime}$ and $u_{3}=$ 0 . Then the stability results follows from the same arguments as made for the previous control input.

Either of the choices for $u_{2}$ implies that $\dot{V}_{3}(z) \leqslant-$ $d_{3} k_{3} W_{3}(z)+d_{3} k_{3} z_{3} u_{3}$ which again implies that $\dot{z}=f_{z}\left(z, u_{3}\right)$ is strictly passive from $u_{3}$ to $z_{3}$ (Khalil, 2002). Hence, it is known that $u_{3}=-\psi\left(t, z_{3}\right)$, where $\psi$ is a time-varying memoryless passive function $\left(z_{3} \psi\left(t, z_{3}\right) \geqslant 0\right)$, makes $z=0$ globally asymptotically stable. Furthermore, it is known that a strictly passive system in feedback interconnection with a strictly passive or output strictly passive and zero state observable system makes the overall system asymptotically stable. This is exploited to generate a control input $u_{3}$ from a system, say $\Sigma_{u 3}$ defined by $\dot{z}_{u 3}=f_{u 3}\left(z_{u 3}, z_{3}\right)$ and $y_{u_{3}}=h\left(z_{u 3}, z_{3}\right)$, where $u_{3}=-y_{u 3}$. Hence, if any of the aforementioned passivity properties holds for $\Sigma_{u 3}$ with a positive definite storage function we have that $\left(z, z_{u 3}\right)=(0,0)$ is asymptotically stable (Khalil, 2002). Assume now that $\Sigma_{u 3}$ is only passive. Considering the function $V\left(z, z_{u}\right)=\left(1 / d_{3} k_{3}\right) V_{3}(z)+V_{s}\left(z_{u 3}\right)$, where $V_{s}$ is the storage function for $\Sigma_{u 3}$, we have that $\dot{V}\left(z, z_{u}\right) \leqslant-d_{3} k_{3} W_{3}(z)$. This implies $\lim _{t \rightarrow \infty} W_{3}(z)=0$ and from (17) it follows that $\lim _{t \rightarrow \infty} z=0$. These passive considerations are summarized in Proposition 3.

\section{Simulations}

The model used for simulation is based on a laboratory installation consisting of a small supercharger driven by a electrical motor, a valve, a plenum volume and in-between ducting. Pressure downstream the compressor is generated in terms of compressor characteristic, $p_{\mathrm{c}}(w, \omega)=\psi_{c}(w, \omega) p_{\mathrm{a}}$, where $p_{\mathrm{a}}$ is the atmospheric ambient pressure and $\psi_{c}(w, \omega)$ is the characteristic shown in the compressor map of Fig. 1. Furthermore, we assume that this pressure is measured ( $p_{\mathrm{c}}$ is implemented as a measurement in the control laws). Numerical values describing the installation are shown in Table 1 , where $(\cdot)_{\mathrm{a}}$ denotes ambient conditions and the speed of sound at ambient conditions is given by $c=\sqrt{\kappa_{\mathrm{a}} R T_{\mathrm{a}}}$.

Simulation starts in a open loop stable operating point $\left(p^{\mathrm{e}}, w^{\mathrm{e}}, \omega^{\mathrm{e}}\right)=\left(1.48 \times 10^{5}, 0.43,3343\right)$. Then a reduction of drive torque drives the system to a new open loop stable operating point $\left(p^{\mathrm{e}}, w^{\mathrm{e}}, \omega^{\mathrm{e}}\right)=\left(1.25 \times 10^{5}, 0.31,2410\right)$ at time $=50 \mathrm{~s}$. Finally, a reduction of the throttle opening drives the system to a open loop unstable operating point

Table 1

Parameters used for simulation

\begin{tabular}{lll}
\hline Ambient & System & Compressor \\
\hline$p_{\mathrm{a}}=101325 \mathrm{~Pa}$ & $V=0.1 \mathrm{~m}^{3}$ & $r=65 \times 10^{-3} \mathrm{~m}$ \\
$T_{\mathrm{a}}=300 \mathrm{~K}$ & $A=5 \times 10^{-3} \mathrm{~m}^{2}$ & $\sigma=0.9$ \\
$R=287 \mathrm{~J} / \mathrm{kg} \mathrm{K}$ & $L=3.6 \mathrm{~m}$ & \\
$\kappa_{\mathrm{a}}=1.4$ & $J=0.039 \mathrm{~kg} \mathrm{~m}^{2}$ & \\
\hline
\end{tabular}




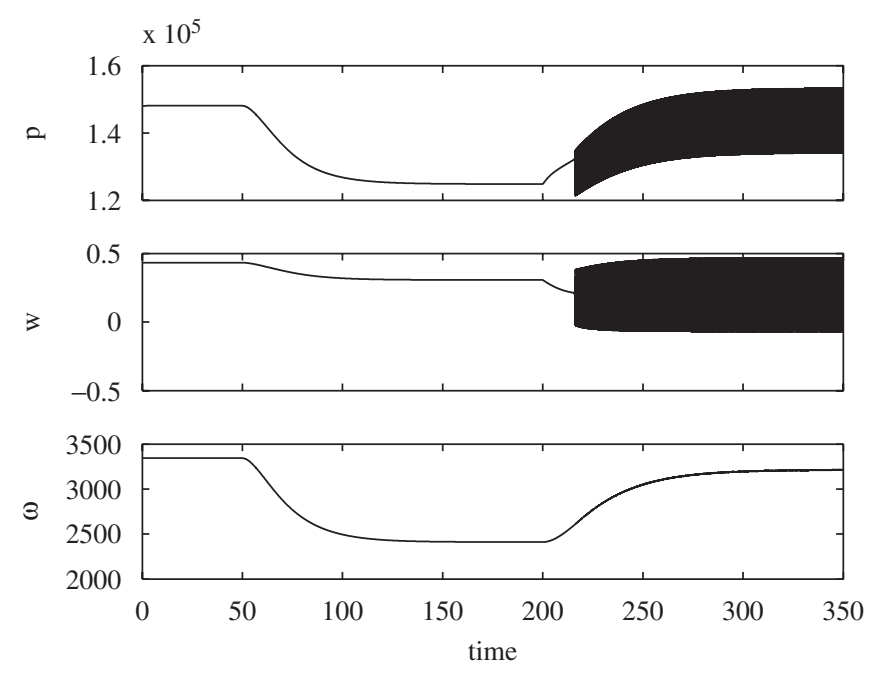

Fig. 3. Open loop simulation.
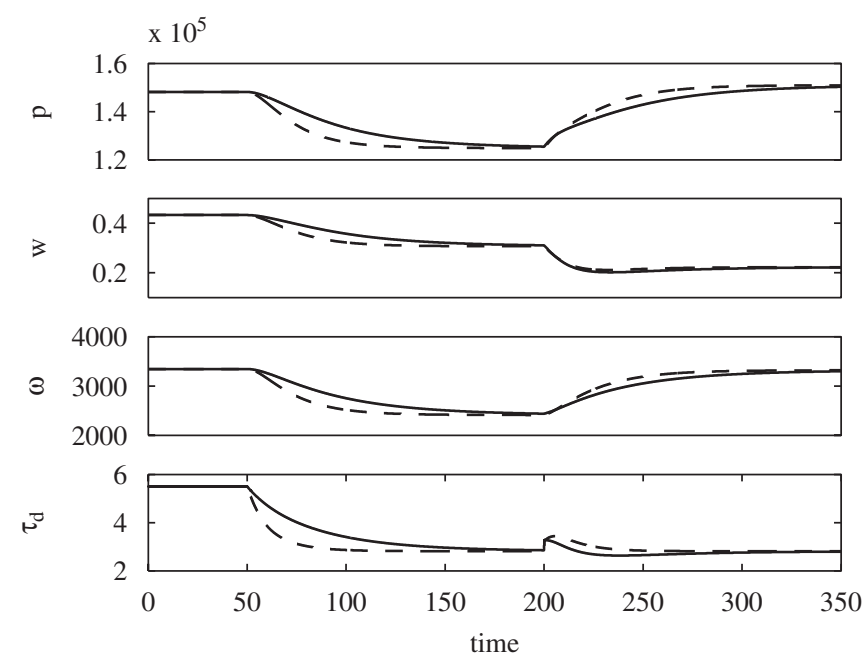

Fig. 4. Solid and dashed lines represent $\tau_{a}$ and $\tau_{b}$, respectively.

$\left(p^{\mathrm{e}}, w^{\mathrm{e}}, \omega^{\mathrm{e}}\right)=\left(1.51 \times 10^{5}, 0.22,3318\right)$ at time $=200 \mathrm{~s}$. All transitions between the operating points are done by a step change through a first order low pass filter with time constant $T_{\mathrm{f}}=10$. This is done to get reasonable magnitudes for the control input during transients. A simulation of the open loop response is shown in Fig. 3, where it can be seen that the system eventually enters surge. By first glance it seems like the impeller speed do not oscillate in surge, but a closer inspection revealed that also this state oscillated with a frequency of approximately $5.6 \mathrm{~Hz}$.

The gain related to the compressor map inequality (6) must be chosen $c_{3}=1250$ to achieve stability. System response for control laws of Proposition 2 are shown in Fig. 4 for $c_{4}=10^{-3}$. From this figure it can be seen that $\tau_{a}$ makes system states converge just in time for change of operating point, while converging somewhat faster for control input $\tau_{b}$. When choosing $c_{4}=0$ the control law $\tau_{a}$ resulted in system states not converging in time for change in equilibrium, whereas $\tau_{b}$ made

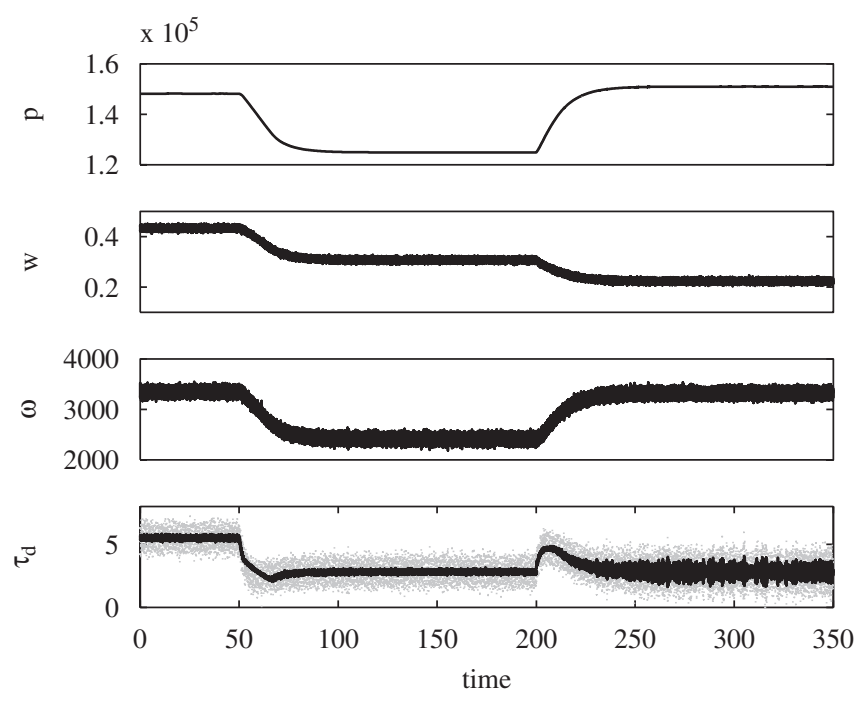

Fig. 5. Black and grey lines represent Propositions 3 and 2, respectively.

the states converge just in time for equilibrium change. When choosing $c_{4}=10^{-2}$ the system states converges relatively fast for both $\tau_{a}$ and $\tau_{b}$. Reducing $c_{3}$ resulted in system states oscillating (system entered surge). All this indicates that $c_{3}$ is playing the role of stabilizing, whereas $c_{4}$ makes the system states converge to the desired equilibrium.

To illustrate on possible choice for the passive control part, white noise of amplitude 0.01 and 100 was added to the measurements of mass flow and impeller speed respectively. The amplitudes of these signals are in the magnitude of $10 \%$ of the measured value. This resulted in the same qualitative time response as in the noise free case, with white noise superimposed on the control input. To suppress the effect of measurement noise on control input, the passive control input was implemented as a first order low pass filter with saturation of the output, $\dot{\tau}=-\left(1 / T_{\text {passive }}\right) \tau+\left(c_{4 \text {,passive }} / T_{\text {passive }}\right) u_{\tau}$ and $\tau_{\text {passive }}=\operatorname{sat}(\tau)$. The various control parameters where chosen as $c_{4}=0, c_{4 \text {,passive }}=10^{-2}, T_{\text {passive }}=0.03$ and a saturation of $1.5 \mathrm{~N} \mathrm{~m}$. The time constant was chosen so that the cut off frequency is approximately that of the surge frequency and $c_{4}$,passive was chosen to replace $c_{4}$. System response is shown in Fig. 5, when using $\tau_{b}$. This figure also shows the corresponding control input when using the control law of Proposition 2. It can be seen that the system converges faster than the simulation shown in Fig. 4. This is due to using a gain, $c_{4}$,passive, which is a decade higher than the gain, $c_{4}$, used in the previous simulation. Furthermore, it can be seen that noise on the control input is considerably reduced when using the passive control law.

\section{Concluding remarks}

The control laws require feedback from all system states, downstream compressor pressure, compressor torque and some model specific constants. Pressure downstream the compressor is used in both $\tau_{a}$ and $\tau_{b}$, whereas the explicit expression for compressor torque is used in $\tau_{a}$ only. Feedback from this 
pressure and torque can be done either by measurement or as functions of mass flow and impeller speed. Furthermore, it is known that measurement of mass flow is difficult, especially in transients. This implies that a mass flow observer must be integrated in the control laws before implementation on an actual plant. One such observer can be found in Bøhagen, Stene, and Gravdahl (2004).

Tuning of control gains should start with choosing $c_{3}$ sufficiently large to achieve stability, since the gain $c_{4}$ can be chosen arbitrarily small in view of Proposition 2 . Then the gain $c_{4}$ can then be increased to improve convergence (alternatively be implemented with saturation as discussed in Section 3). Moreover, it is believed that best results is obtained by using Proposition 3 and choosing $c_{3}$ as small as possible so that stability is achieved. Then use a dynamic part introduced by $\tau_{\text {passive }}$ for performance. The motivation for this is found in disturbances, since a dynamic controller can offer low pass filtering in addition to gain. Furthermore, a dynamic control law can also offer integral action on its error variable which might improve steady state performance in the presence of model uncertainty.

When implementing the control law with models of downstream compressor pressure and compressor torque, these implementations are subject to model uncertainties. The models enters the control law when using $u_{2}$ to cancel all terms in (16). Since the cancellations are in the span of the actual control input $u_{2}$ (matching condition), it is a simple exercise to extend the control laws with nonlinear damping to compensate for model uncertainties. The same arguments holds for extending to adaptive control laws for $A J / L$ and $\sigma r^{2}$, in the case when these constants are unknown or slowly varying.

For the current simulations, the gain $c_{3}$ had to chosen relatively high. Therefore no measurement noise was added to $p$ or $p_{\mathrm{c}}$ because this resulted in unrealistic control inputs when operating left of the surge line. The compressor characteristic was implemented with third order polynomial approximations of mass flow and impeller speed Willems (2000), based on data from the open loop stable region and theoretical points for zero mass flow. This implies that the theoretical compressor characteristic is derived without the explicit knowledge of compressor behavior in the open loop unstable region. Hence, the value needed for $c_{3}$ for an actual system should be determined experimentally.

\section{References}

Bøhagen, B., \& Gravdahl, J. T. (2005). Active control of compression systems using drive torque; a backstepping approach. In Procedings of the 44th IEEE conference on decision and control, December.

Bøhagen, B., \& Gravdahl, J. T. (2006). Active surge control using drive torque: dynamic control laws. In Proceedings of the 45th IEEE conference on decision and control, December.

Bøhagen, B., Stene, O., \& Gravdahl, J. T. (2004). A ges mass flow observer for compression systems: Design and experiments. In Proceedings of the 2004 american control conference, June.
Epstein, A. H., Ffowcs Williams, J. E., \& Greitzer, E. M. (1989). Active suppression of aerodynamic instabilities in turbomachines. Journal of Propulsion and Power, 5(2), 204-211.

Fink, D. A., Cumpsty, N. A., \& Greitzer, E. M. (1992). Surge dynamics in free-spool centrifugal compressor system. Journal of Turbomachinery, 114, 321-332.

Gravdahl, J. T., \& Egeland, O. (1999). Compressor surge and rotating stall: Modeling and control. Advances in industrial control. Berlin: Springer.

Gravdahl, J. T., Egeland, O., \& Vatland, S. O. (2002). Drive torque actuation in active surge control of centrifugal compressors. Automatica, 38(11), 1881-1893.

Gravdahl, J. T., Willems, F., de Jager, B., \& Egeland, O. (2004). Modeling of surge in variable speed centrifugal compressors: Experimental validation. AIAA Journal of Propulsion and Power, 20(5), 849-857.

Greitzer, E. M. (1976). Surge and rotating stall in axial flow compressors, part i: Theoretical compression system model. Journal of Engineering for Power, 98, 190-198.

Khalil, H. K. (2002). Nonlinear system. (3rd ed.), Englewood Cliffs, NJ: Prentice-Hall.

Krstić, M., Fontaine, D., Kokotović, P. V., \& Paduano, J. D. (1998). Useful nonlinearities and global stabilization of bifurcations in a model of jet engine surge and stall. IEEE Transactions on Automatic Control, 43(12), $1739-1745$.

Krstić, M., Kanellakopoulos, I., \& Kokotović, P. V. (1995). Nonlinear and adaptive control design. New York: Wiley.

van de Wal, M. (1998). Selection of inputs and outputs for control. Ph.D. thesis, Eindhoven University of Technology.

Willems, F. (2000). Modeling and bounded feedback stabilization of centrifugal compressor surge. Ph.D. thesis, Eindhoven University of Technology, Eindhoven, The Netherlands.

Willems, F., \& de Jager, B. (1999). Modeling and control of compressor flow instabilities. IEEE Control Systems Magazine, 19(5), 8-18.

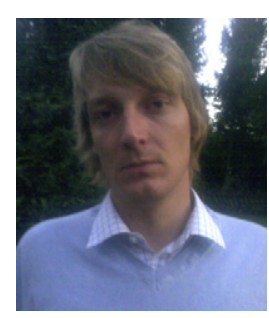

Bjørnar Bøhagen received the siv.ing. and the $\mathrm{Ph} . \mathrm{D}$. degrees in Engineering Cybernetics from the Norwegian University of Science and Technology in 2002 and 2007, respectively. He is currently with $\mathrm{ABB}$.

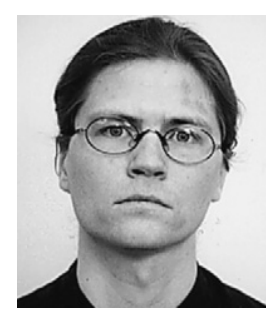

Jan Tommy Gravdahl received the siv.ing. and the dr.ing. degrees in Engineering Cybernetics from the Norwegian University of Science and Technology in 1994 and 1998, respectively. $\mathrm{He}$ is currently an Associate Professor at the Department of Engineering Cybernetics at the Norwegian University of Science and Technology, Trondheim, Norway. He has received the IEEE Transactions on Control Systems Technology Outstanding Paper Award for the year 2000, and is author of Compressor Surge and Rotating Stall. Modeling and Control (Springer 1999) and Modeling and Simulation for Automatic Control (Marine Cybernetics 2002), which are co-authored with Olav Egeland. His research interests include modeling and nonlinear control of mechanical systems with application to compression systems and satellites. 